\title{
Pathologic and biologic response to preoperative endocrine therapy in patients with ER-positive ductal carcinoma in situ Yunn-Yi Chen ${ }^{1}$, Sandy DeVries ${ }^{2}$, Joseph Anderson ${ }^{2}$, Juan Lessing ${ }^{3}$, Rebecca Swain ${ }^{1}$, Koei Chin ${ }^{2}$, Veronica Shim ${ }^{5}$, Laura J Esserman ${ }^{3,4}$, Frederic M Waldman ${ }^{2,3}$ and E Shelley Hwang*3,4
}

\begin{abstract}
Address: ${ }^{1}$ Department of Pathology, University of California San Francisco, San Francisco, USA, ${ }^{2}$ Department of Laboratory Medicine, University of California San Francisco, San Francisco, USA, ${ }^{3}$ Department of Surgery, University of California San Francisco, San Francisco, USA, ${ }^{4}$ Helen Diller Family Comprehensive Cancer Center, University of California, San Francisco, USA and ${ }^{5}$ Department of Surgery, Kaiser Permanente, Oakland, USA

Email: Yunn-Yi Chen - Yunn-Yi.Chen@ucsf.edu; Sandy DeVries - DeVriesS@labmed2.ucsf.edu;

Joseph Anderson - janderson@genomichealth.com; Juan Lessing - juan.lessing@ucsf.edu; Rebecca Swain - Rebecca.swain@ucsf.edu; Koei Chin - ChinK@labmed2.ucsf.edu; Veronica Shim - veronica.shim@kp.org; Laura J Esserman - laura.esserman@ucsfmedctr.org; Frederic M Waldman - WaldmanF@labmed2.ucsf.edu; E Shelley Hwang* - shelley.hwang@ucsfmedctr.org

* Corresponding author
\end{abstract}

Published: 18 August 2009

BMC Cancer 2009, 9:285 doi:10.1 186/147|-2407-9-285

This article is available from: http://www.biomedcentral.com/147/-2407/9/285

(C) 2009 Chen et al; licensee BioMed Central Ltd.

This is an Open Access article distributed under the terms of the Creative Commons Attribution License (http://creativecommons.org/licenses/by/2.0), which permits unrestricted use, distribution, and reproduction in any medium, provided the original work is properly cited.

\begin{abstract}
Background: Endocrine therapy is commonly recommended in the adjuvant setting for patients as treatment for ductal carcinoma in situ (DCIS). However, it is unknown whether a neoadjuvant (preoperative) anti-estrogen approach to DCIS results in any biological change. This study was undertaken to investigate the pathologic and biomarker changes in DCIS following neoadjuvant endocrine therapy compared to a group of patients who did not undergo preoperative antiestrogenic treatment to determine whether such treatment results in detectable histologic alterations.
\end{abstract}

Methods: Patients $(n=23)$ diagnosed with ER-positive pure DCIS by stereotactic core biopsy were enrolled in a trial of neoadjuvant anti-estrogen therapy followed by definitive excision. Patients on hormone replacement therapy, with palpable masses, or with histologic or clinical suspicion of invasion were excluded. Premenopausal women were treated with tamoxifen and postmenopausal women were treated with letrozole. Pathologic markers of proliferation, inflammation, and apoptosis were evaluated at baseline and at three months.

Biomarker changes were compared to a cohort of patients who had not received preoperative treatment.

Results: Median age of the cohort was 53 years (range 38-78); 14 were premenopausal. Following treatment, predominant morphologic changes included increased multinucleated histiocytes and degenerated cells, decreased duct extension, and prominent periductal fibrosis. Two postmenopausal patients had ADH only with no residual DCIS at excision. Postmenopausal women on letrozole had significant reduction of PR, and Ki67 as well as increase in CD68positive cells. For premenopausal women on tamoxifen treatment, the only significant change was increase in CD68. No change in cleaved caspase 3 was found. Two patients had invasive cancer at surgery.

Conclusion: Preoperative therapy for DCIS is associated with significant pathologic alterations. These changes may be clinically significant. Further work is needed to identify which women may be the best candidates for such treatment for DCIS, and whether best responders may safely avoid surgical intervention.

Trial Registration: ClinicalTrials.gov NCT00290745 


\section{Background}

Ductal carcinoma in situ (DCIS) was diagnosed in over 60,000 women in the United States in 2008[1]. The incidence of DCIS has risen almost 5-fold over the last 15 years, and now represents $25-30 \%$ of all mammographically detected breast cancers[2,3]. Moreover, studies suggest that the undetected disease reservoir of DCIS could be even larger. Autopsy series in women dying of causes other than breast cancer show that over $10 \%$ of whole breast specimens may harbor DCIS not previously recognized [4-6]. Since DCIS is rarely palpable, mammography is the primary mode of detection. As mammographic screening has become more sensitive and widespread, more clinically occult preinvasive disease continues to be detected.

The current treatment of DCIS is based on a presumption that DCIS is a non-obligate precursor of invasive breast cancer. There is a paucity of natural history studies since DCIS is generally surgically resected upon diagnosis. The few retrospective reports of women who had biopsies that were assumed to be benign but on later review were found to have DCIS report a $20-50 \%$ risk of invasive cancer in the twenty years after biopsy [7-12]. Without validated measures with which to stratify future risk for invasive cancer, the current goal of all DCIS treatment is prevention of cancer progression through surgery, radiation, hormonal therapy, or a combination of these modalities[7]. In fact, despite the 99\% survival rate from DCIS, these aggressive treatments are not much different than those recommended for the invasive cancers these interventions are aimed to prevent. Thus, current therapy for DCIS may represent overtreatment for many women who may never progress to invasive cancer. Nevertheless, expectant management alone is not currently considered an acceptable alternative for most women, due to fear of invasive progression.

In prospective randomized trials, adjuvant tamoxifen and aromatase inhibitor (AI) have both been associated with significantly reduced risk of contralateral invasive breast cancer [13-16]. One possible explanation for this observation is that endocrine therapy may prevent progression of in situ to invasive disease. If indeed the risk of DCIS progression could be reduced with primary medical therapy alone, some women with DCIS could potentially avoid the morbidity of surgery and radiation as well as derive benefit from contralateral risk reduction. Tamoxifen is already offered as adjuvant treatment for DCIS, based on data from a prospective placebo-controlled trial in women undergoing lumpectomy and radiation showing a benefit in DFS which favored tamoxifen[14]. Ongoing clinical trials are studying the role of AI in both the prevention and adjuvant settings for DCIS. However, the bio- logic impact of endocrine therapy directly on DCIS lesions themselves remains largely unexplored.

To our knowledge, this is the first report examining the biologic changes that accompany preoperative endocrine treatment of ER-positive pure DCIS. Our aim was to determine whether a 3-month course of preoperative endocrine therapy for DCIS could result in measurable histologic and immunohistochemical changes when compared to baseline assessments made on initial diagnostic core biopsy. To reduce possible systematic bias in immunohistochemistry (IHC) scoring arising from differences in tissue fixation of core biopsy versus surgical excision specimens, we also performed a matched analysis of IHC changes on core biopsy compared to surgical excision on controls who did not undergo preoperative treatment.

\section{Methods \\ Treated Cases}

Patients treated at the University of California San Francisco Comprehensive Cancer Center between 2003 and 2007 were offered participation in an investigator-initiated clinical trial studying the effect of preoperative endocrine therapy in DCIS. The study was reviewed and approved by the UCSF IRB in compliance with the Helsinki Declaration (CHR approval H10367-19435), and written informed consent from all participants was documented. Patients diagnosed with pure DCIS by stereotactic core needle biopsy of microcalcifications were recruited to a trial of 3 months of neoadjuvant endocrine therapy. Patients with current hormone replacement use, palpable masses, hormone receptor-negative DCIS, or clinical suspicion of invasion were excluded from study participation.

Premenopausal patients were treated with tamoxifen (20 mg PO QD), while postmenopausal patients were treated with letrozole (2.5 mg PO QD). Definitive excision consisting of either lumpectomy or mastectomy was planned per study protocol at the completion of three months of preoperative therapy.

\section{Untreated Controls}

Patients undergoing surgery for DCIS at our institution between 2003 and 2006 who consented to have tissue banked for research were identified. Those with invasive cancer and those for whom diagnostic core biopsy blocks or surgical blocks were not available were excluded. Eighteen consecutive cases were evaluable for all biomarkers assessed.

\section{Histopathology and Biomarker Assessment}

Hematoxylin and eosin (H\&E)-stained sections from all pre-treatment and post-treatment biopsies were reviewed 
by the study pathologist (YC). Diagnosis was rendered according to the criteria established by Page et al[17] and the WHO classification system[18]. Grading was based on the evaluation of cytonuclear features and divided into three grades ( 1 to 3 ). Other features of DCIS were also recorded including architectural pattern, presence and type of necrosis, microcalcifications, associated foamy macrophages and multinucleated giant cells.

Immunohistochemistry was performed on formalinfixed, paraffin-embedded tissue sections using the streptavidin-biotin peroxidase method. Antibodies and dilutions used were: ER (DAKO, Carpinteria, CA) at 1:400 dilution; PR (Novocastra) at 1:25; HER2 (Zymed) at 1:200; Ki-67 (DAKO) at 1:100; CD68 (DAKO) at 1:100; and cleaved caspase 3 (Cell Signaling, Beverly, MA) at 1:200.

Antigen retrieval was achieved using either heat-induced epitope retrieval in $10 \mathrm{mM}$ citrate buffer at $\mathrm{pH} 6.0$ (for ER, PR, CD68, and caspase 3), incubation with Ficin (Zymed) at $37^{\circ} \mathrm{C}$ (for HER2), or $0.01 \%$ trypsin digestion followed by heat treatment in $10 \mathrm{mM}$ citrate buffer (for Ki-67). Slides were blocked in $3 \% \mathrm{H}_{2} \mathrm{O}_{2}$ and then incubated with the primary antibodies. HER2 gene amplification was determined by FISH analysis using the PathVysion HER2 DNA Probe Kit (Vysis Inc., Downer's Grove, IL) according to manufacturer recommendations.

In order to minimize the impact of IHC staining variability in the analysis, specific care was taken to ensure that matched pretreatment and postreatment specimens were stained in the same run.

\section{Scoring of Biomarkers}

All immunostains were evaluated by two pathologists (YC and RS) and scored randomly so that pre- and post-treatment samples were evaluated independently for both cases and controls. The scoring was perfomed without knowledge of other immunostains from the same specimen. Pathologists were also blinded to the scoring of the paired pre- or post-treatment sample.

$E R$ and $P R$ were scored by evaluating the percentage and intensity of stained tumor nuclei (H-score) as previously described $[19,20]$. Staining intensity ranged from 0 to $3+$, with 0 representing no staining, $1+$ weak staining, $2+$ moderate staining, and $3+$ strong staining. Percentages of positive tumor cells in each staining intensity category were recorded. Results of ER and PR were expressed as the H-score where: H-score $=(1 \times \% 1+)+(2 \times \% 2+)+(3 \times$ $\% 3+)$.

HER2 was scored by criteria established by the HercepTest (DAKO), using a 0-3 scale, based on staining intensity of tumor cells. Staining intensities 0 and 1 were considered negative; intensity 2 indeterminate, and intensity 3 positive for HER2 protein overexpression. FISH analysis was performed on all cases with staining intensity $2-3$ by immunohistochemistry, and cases showing a ratio of HER2:centromere 17 copy number greater than 2.0 were considered positive for gene amplification.

Ki-67 staining was used to establish a proliferation index. Slides were first scanned at low-power magnification to select DCIS foci with highest mitotic activity. Where possible, at least 500 tumor cells were counted in these mitotically active areas. Proliferation index was obtained by the percentage of tumor nuclei that were labeled by Ki-67.

CD68 staining was used to identify DCIS infiltrating macrophages. Macrophage density was recorded by scanning at low-power magnification to choose DCIS foci with highest concentration of CD68 positive cells. The number of intraductal CD68 positive macrophages was counted in three high-power fields (hpf, 400×). The macrophage density was expressed as the mean number of macrophages per hpf.

Cleaved caspase 3 staining was used to evaluate activation of apoptosis, and was interpreted as positive in tumor cells with strong granular staining in the cell. Where possible, a minimum of 500 tumor cells was scored in each sample. Only predominantly intact cells were counted. The results were expressed as the percentage of tumor cells expressing cleaved caspase 3.

\section{Statistics}

A non-parametric rank-sum test for non-normalized distribution of data was used to compare changes in IHC markers between cases and controls, as well as between baseline and treated cases. Significance was established at a $p$ value of $<0.05$.

\section{Results \\ Patient Characteristics}

From 2003 to 2007, 23 patients completed the study protocol with both pre- and post-treatment materials available for analysis. Overall, the treatment was well tolerated. One patient (H-24) was discontinued from the study; she was taken off tamoxifen on day 31 due to an arterial embolic event.

Two premenopausal patients declined excision and chose to continue the endocrine treatment. However, follow-up post-treatment core needle biopsies were performed on these two patients and were compared to pre-treatment samples. The remaining patients underwent definitive surgical excision following preoperative endocrine ther- 
Table I: Characteristics of Study Population

\begin{tabular}{lccc}
\hline & & \multicolumn{2}{c}{ Cases } \\
\cline { 3 - 4 } & Controls & Postmenopausal & Premenopausal \\
\hline $\mathrm{N}$ & 18 & 9 & 14 \\
Age at diagnosis $(\mathrm{y})$ & 54 & 61 & 46 \\
$\begin{array}{l}\text { DCIS grade } \\
\text { low }\end{array}$ & & & \\
$\quad$ intermediate & 7 & 0 & 1 \\
$\quad$ high & 9 & 5 & 6 \\
$\begin{array}{l}\text { Type of surgery } \\
\text { lumpectomy } \\
\text { mastectomy }\end{array}$ & 16 & 4 & 7 \\
none & 2 & 8 & 7 \\
\hline
\end{tabular}

apy. Table 1 and Additional File 1http://wald man.ucsf.edu/Waldman.Primary.Data.html summarize the clinical characteristics of the study population.

\section{Pathologic Features}

The baseline pre-treatment core biopsies showed a range of DCIS nuclear grade with 12/23 patients diagnosed with non-high grade disease (Table 1 ). Comparison of tumor grade between pre- and post-treatment specimens showed that the nuclear grade remained the same in 14 cases, changed to lower grade in five, and became higher grade in two cases (Table 2; Additional File 2: http://wald man.ucsf.edu/Waldman.Primary.Data.html).

Extent of surgery was guided by mammographic extent of calcifications. In two patients, post-treatment surgical specimens demonstrated atypical ductal hyperplasia $(\mathrm{ADH})$ only. These patients had residual mammographically visible calcifications following core biopsy, with calcifications confirmed pathologically on the posttreatment surgical specimen, but no DCIS was seen associated with microcalcifications following treatment.

DCIS was identified in 21 patients following endocrine treatment. Morphologically, the post-treatment samples were less distended and demonstrated increased periductal fibrosis and inflammation when compared to the pretreatment biopsies (Figures 1a, 1b). Treated samples also had more pronounced multinucleated histiocytes and degenerated cells within DCIS and ADH. In addition to multinucleated histiocytes and degenerated cells, the $\mathrm{ADH}$ in post-treatment samples demonstrated microcalcifications similar to those noted in the pre-treatment DCIS or adjacent post-treatment DCIS, suggesting some of the $\mathrm{ADH}$ could represent treated DCIS but with a lesser degree

Table 2: Comparison of histologic diagnosis at baseline and following neoadjuvant anti-estrogenic therapy

\begin{tabular}{|c|c|c|c|c|}
\hline ID & Age at diagnosis & Menopausal status & Baseline diagnosis ${ }^{\prime}$ & Post-treatment diagnosis \\
\hline $\mathrm{H}-02$ & 56 & post & G2-3 DCIS, LCIS & G2 DCIS, G2 IDC (1.2 cm), LCIS \\
\hline $\mathrm{H}-04^{2}$ & 41 & pre & G2-3 DCIS & G2-3 DCIS \\
\hline $\mathrm{H}-07$ & 48 & pre & G2 DCIS & G2 DCIS \\
\hline $\mathrm{H}-14$ & 65 & post & GI-2 DCIS & $\mathrm{ADH}$ \\
\hline $\mathrm{H}-\mathrm{I} 5$ & 49 & pre & G2 DCIS & GI-2 DCIS, ADH \\
\hline $\mathrm{H}-16^{2}$ & 60 & post & G2 DCIS & G3 DCIS, G2 IDC $(1.8 \mathrm{~cm})$ \\
\hline $\mathrm{H}-17$ & 45 & pre & GI DCIS, ADH, FEA & GI DCIS, ADH, FEA \\
\hline $\mathrm{H}-18$ & 53 & pre & G3 DCIS & G3 DCIS \\
\hline $\mathrm{H}-19$ & 47 & pre & G2 DCIS, ADH & GI DCIS \\
\hline $\mathrm{H}-20$ & 55 & post & GI-2 DCIS & G2 DCIS \\
\hline $\mathrm{H}-2 \mathrm{I}$ & 52 & pre & G2 DCIS & G2 DCIS \\
\hline $\mathrm{H}-22$ & 65 & post & GI-2 DCIS & GI DCIS, ADH \\
\hline $\mathrm{H}-23$ & 44 & post & G2 DCIS & GI DCIS \\
\hline $\mathrm{H}-24$ & 42 & pre & G2 DCIS, FEA & G2-3 DCIS, FEA \\
\hline $\mathrm{H}-27$ & 43 & pre & G2-3 DCIS & G2 DCIS \\
\hline $\mathrm{H}-29$ & 52 & post & G3 DCIS & $\mathrm{ADH}$ \\
\hline $\mathrm{H}-30^{2}$ & 78 & post & G3 DCIS & G3 DCIS \\
\hline $\mathrm{H}-3 \mathrm{I}^{2}$ & 52 & post & G2-3 DCIS & G2-3 DCIS \\
\hline $\mathrm{H}-33$ & 41 & pre & G2-3 DCIS & G2-3 DCIS \\
\hline $\mathrm{H}-34$ & 42 & pre & G2 DCIS & GI-2 DCIS \\
\hline $\mathrm{H}-35$ & 68 & post & G3 DCIS & G3 DCIS \\
\hline $\mathrm{H}-36$ & 44 & pre & G2-3 DCIS & G2-3 DCIS \\
\hline $\mathrm{H}-38^{2}$ & 46 & pre & G2-3 DCIS & G2-3 DCIS \\
\hline
\end{tabular}

Iabbreviation: G: grade; DCIS: ductal carcinoma in situ; IDC: invasive ductal carcinoma; LCIS: lobular carcinoma in situ; ADH: atypical ductal hyperplasia; FEA: flat epithelial hyperplasia.

2Her2 IHC 3+ 

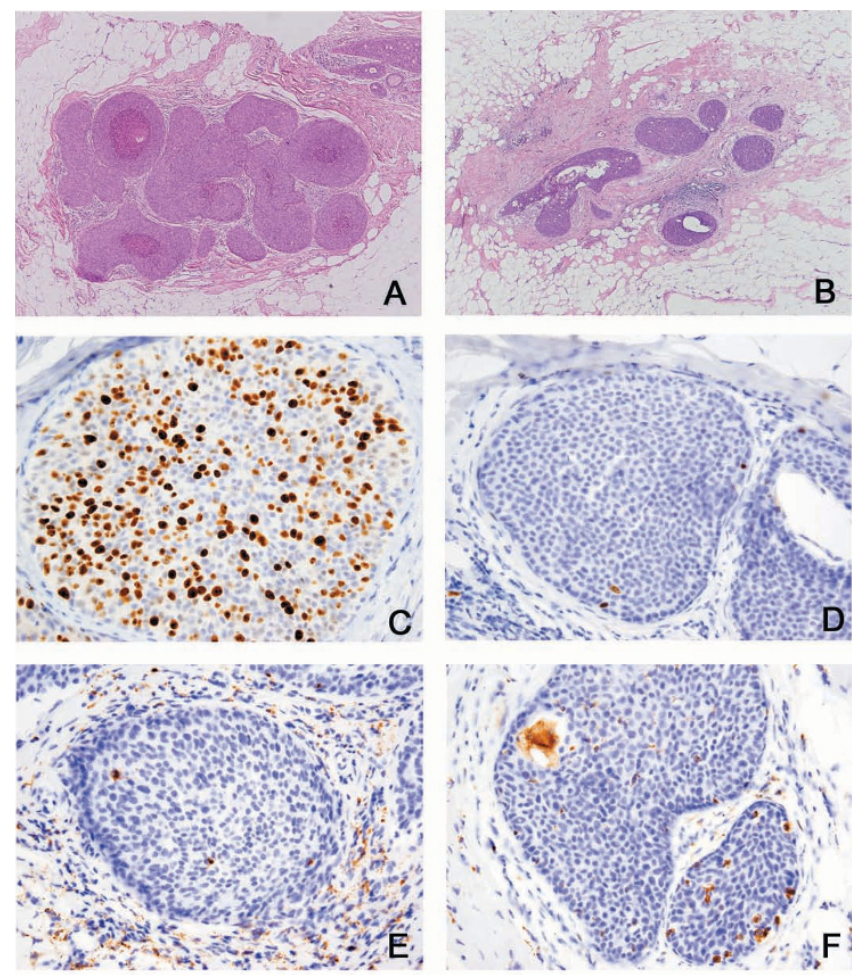

Figure I

Histology of baseline (A, C, E) and endocrine-treated (B, D, F) DCIS from patient $\mathbf{H}-20$ (magnification: I 00x). A, B: H\&E stain of baseline (A) and treated (B) samples. The treated DCIS is less distended and demonstrates increased periductal sclerosis and inflammation compared to baseline. C, D: Ki67; reduction in Ki-67 after treatment compared to baseline; E, F: CD68 (inset: 400x); increased CD68positive macrophages after treatment compared to baseline.

of cytoarchitectural features for diagnosis of DCIS. Two postmenopausal patients were also found to have intermediate grade, ER-positive invasive ductal carcinoma, measuring 1.2 and $1.8 \mathrm{~cm}$ respectively.

\section{Biomarker Expression}

The range of IHC staining for ER, Ki67, CD68, and caspase 3 for all three grades of DCIS is illustrated in Additional File 3. Changes in DCIS IHC expression from baseline to treated specimens are summarized in Table 3 . The magnitude of change in treated cases and untreated controls were compared.

\section{$E R$}

Hormone treatment resulted in a reduction of ER expression as demonstrated by the ER H-score. Although there was downregulation of ER expression, all DCIS lesions remained ER-positive. Reduction of ER staining was modest in the majority of the patients but at least $50 \%$ in 9 of 23 patients. This reduction was most pronounced among the postmenopausal women treated with letrozole, where it approached statistical significance.

$P R$

Endocrine treatment resulted in a marked reduction in PR expression. Three cases completely lost PR staining after treatment $(\mathrm{H}$-score $=0)$ while all the other cases remained PR-positive. Nine of 23 cases had at least 50\% downregulation in percentage of tumor cells staining positive for PR. This reduction in PR expression was significant among all cases, but stratified analysis showed that the reduction in PR was limited to the postmenopausal group treated with letrozole.

\section{Ki-67}

A significant reduction in Ki-67 labeling index was observed after endocrine therapy among both pre- and post-menopausal patients (Figures 1c, 1d). This change was significant when compared to untreated controls. DCIS nuclear grade was correlated with mean pre-treatment Ki67 (grade 1: $7.7 \%$; grade 2: $17.4 \%$; grade 3 : $23.5 \%$ ), although this finding was not statistically significant $(\mathrm{p}=0.09)$. Notably, grade 3 DCIS showed the greatest mean reduction of Ki67 with treatment (13.3\%), compared to grade 1 and grade 2 DCIS (6.5\% and $6.1 \%$ respectively). The mean reduction in Ki-67 in postmenopausal cases was greater than that observed in premenopausal cases (Figures 2a, 2b).

\section{Macrophage density}

Analysis of macrophage density by CD68 showed that endocrine therapy was associated with a significant increase of macrophages within the lesional ducts compared to controls (Table 3; Figures 2c, 2d). All except two cases demonstrated an increase in macrophage density in post-treatment specimens. The change in macrophage density was correlated with the morphologic findings of many foamy macrophages and multinucleated giant cells identified in the post-treatment samples (Figures 1e, 1f).

HER2-neu

Of the 23 cases, five showed both HER2 protein overexpression (staining intensity 3 on immunohistochemistry) and/or HER2 gene amplification (HER2 gene to centromere 17 ratio $>2$ ). Neoadjuvant endocrine therapy did not alter the HER2 status in any of the DCIS lesions. Interestingly, the reduction in Ki-67 did not differ significantly between HER2-positive and HER2-negative cases.

\section{Cleaved caspase 3}

There was no significant treatment effect on apoptosis as measured by the cleaved caspase 3 assay. Among those treated with endocrine therapy, twelve cases showed increased expression of cleaved caspase 3, although these changes were of small magnitude (mean change 0.1, range -6.5 to 6 ). 


\section{Discussion}

The aim of our study was to determine the effects of preoperative endocrine therapy on morphology and biomarker expression in DCIS. We found that administration of anti-estrogenic treatment for DCIS resulted in marked morphologic changes, decreased proliferation, and protein expression changes. Changes were compared to controls who did not undergo preoperative treatment, confirming that our findings were associated with intervening therapy rather than differences in tissue fixation between core biopsy and surgical specimens. Endocrine therapy also increased macrophage density within DCIS ducts. However, a significant change in apoptosis as measured by cleaved caspase 3 was not observed, suggesting that apoptosis was not significantly affected by this short course of treatment.

Clinical trials of neaodjuvant endocrine therapy in estrogen-positive invasive breast cancer have demonstrated a clear clinical objective response of at least 35\% for both tamoxifen and aromatase inhibitors at 3 or 4 months [2123]. If hormone therapy also induces regression in DCIS, alterations in morphology of DCIS would have been expected. In the present study, most cases treated with neoadjuvant therapy showed morphologic changes in the DCIS. These included a decrease in duct distention, an increase in periductal sclerosis and scattered degenerated cells, as well as an increase in macrophage infiltrate. These morphologic changes are compatible with treatment effect and may represent regression of DCIS. Furthermore, two of the 23 cases treated with endocrine therapy demonstrated only ADH without residual DCIS in the posttreatment excision specimens. We cannot entirely exclude the possibility that all DCIS was removed during the initial core biopsy for these two patients. However, the ADH seen in the post-treatment specimens was present immediately adjacent to biopsy site changes, demonstrated similar microcalcifications as those observed in the original DCIS, and showed similar cytologic features but less developed architecture as in the corresponding pre-treat- ment DCIS. Furthermore, degenerating epithelial cells and multinucleated giant cells were noted within the ADH ducts. Therefore, the morphologic features of these posttreatment ADH suggest that they may represent altered DCIS associated with treatment.

The anti-tumor effect of targeted endocrine therapy could be attributed to decreased proliferation and/or increased apoptosis at the cellular level. In this study, neoadjuvant endocrine therapy reduced cellular proliferation in DCIS cells, as measured by reduction in Ki-67. Our findings are in agreement with data reported by Boland et al. which showed a significant reduction in the percentage of proliferating cells in ER-positive DCIS following estrogen withdrawal[24]. Previous studies have also demonstrated an antiproliferative effect of tamoxifen and aromatase inhibitors in invasive breast cancer [25-31]. Most recently, in a study evaluating the DCIS component in prospective randomized window trial of letrozole versus anastrozole for ER-positive postmenopausal invasive cancer, a significant drop in Ki67 was seen for both groups in the DCIS component of the tumor[32]. In aggregate, these studies confirm that endocrine therapy effectively reduces proliferation in DCIS.

However, endocrine therapy did not have a discernible effect on apoptosis as measured by the cleaved caspase 3 assay. The lack of an observed apoptotic effect could be due to the limited sample size, or other apoptotic pathways not detected by the caspase 3 assay. Alternatively the drug concentrations at the DCIS site after oral tamoxifen or letrozole may have been below the levels required for activation of such programmed cell death pathways. Previous studies have shown conflicting results on the association between endocrine treatment and apoptosis. While in vitro[33,34], animal model[35] and one clinical[36] study demonstrated activation of apoptosis by endocrine therapy, other clinical trials on invasive breast cancer failed to show increased apoptosis by tamoxifen, raloxifene, or letrozole $[31,37,38]$. Further studies are

Table 3: Change in biomarkers with anti-estrogenic treatment

\begin{tabular}{|c|c|c|c|c|c|c|c|}
\hline \multirow[b]{2}{*}{ Biomarker } & \multirow{2}{*}{$\begin{array}{c}\text { Controls }(n=18) \\
\text { Mean changel }\end{array}$} & \multicolumn{2}{|c|}{ All Cases $(n=23)$} & \multicolumn{2}{|c|}{ Postmenopausal Cases $(n=9)$} & \multicolumn{2}{|c|}{ Premenopausal Cases $(n=14)$} \\
\hline & & Mean change & $p^{2}$ & Mean change & $p$ & Mean change & $p$ \\
\hline ER $(\mathrm{H} \text {-score })^{3}$ & $-55.5 \pm 22.2$ & $-92.1 \pm 23.7$ & 0.21 & $-113.8 \pm 26.2$ & 0.07 & $-78.2 \pm 35.4$ & 0.62 \\
\hline PR (H-score) ${ }^{3}$ & $-38.8 \pm 18.3$ & $-110.1 \pm 18.7$ & 0.03 & $-169.2 \pm 18.8$ & 0.001 & $-72.1 \pm 22.0$ & 0.40 \\
\hline $\mathrm{Ki}-67^{4}$ & $-3.2 \pm 1.7$ & $-10.2 \pm 1.8$ & 0.007 & $-14.4 \pm 3.6$ & 0.013 & $-7.5 \pm 1.6$ & 0.037 \\
\hline CD684 & $-0.06 \pm 1.7$ & $34.1 \pm 8.3$ & $<0.0001$ & $29.4 \pm 5.8$ & $<0.0001$ & $37.2 \pm 13.2$ & 0.002 \\
\hline caspase $3^{5}$ & $0.8 \pm 0.7$ & $0.1 \pm 0.4$ & 0.15 & $-0.1 \pm 0.7$ & 0.13 & $0.2 \pm 0.5$ & 0.33 \\
\hline
\end{tabular}

'Data shows mean increase (positive values) or mean decrease (negative values) in quantitative immunohistochemical staining between diagnostic core biopsy and surgical excision. Cases were treated with neoadjuvant anti-estrogenic therapy; controls did not undergo neoadjuvant therapy.

${ }^{2}$ Rank-sum test, controls as referent group.

${ }^{3} \mathrm{H}$-score is weighted measure of intensity and percentage of positive cells (see Methods).

${ }^{4}$ Percent of cells staining positive.

${ }^{5}$ Number of CD68-positive macrophages per high power field within DCIS ducts. 

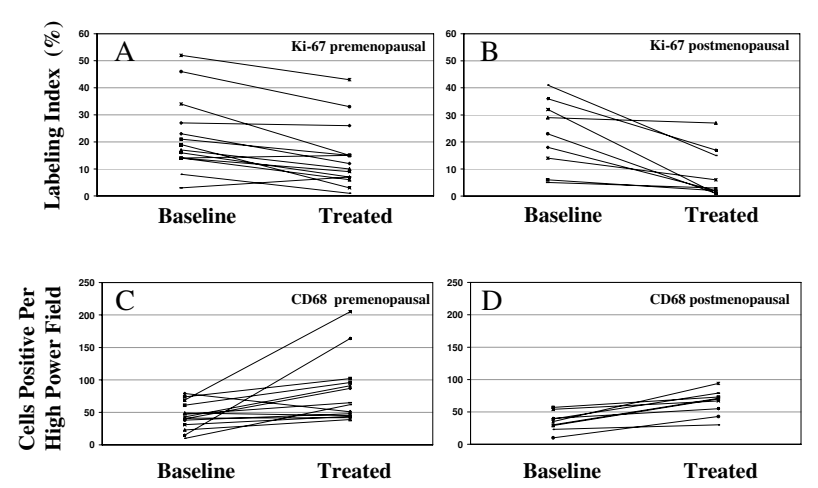

Figure 2

Changes in Ki67 labeling index (A, B) and CD68-positive macrophage count $(C, D)$ between baseline and treated DCIS. There was significant reduction in $\mathrm{Ki} 67$ in premenopausal cases $(p=0.04)$ and in postmenopausal cases $(p=0.01)$. Similarly, CD68-positive macrophage density increased in both premenopausal $(p=0.002)$ and in postmenopausal cases $(p<0.000 I)$. Significance was determined by the rank-sum test comparing treated to baseline values.

required to elucidate the effect on apoptotic activity by endocrine therapy in DCIS.

Short-term treatment with AI resulted in downregulation of ER and PR expression in the DCIS cells, an effect not seen with tamoxifen treatment. This is consistent with studies in invasive breast cancer which also demonstrated changes in ER and PR expression after endocrine therapy $[26,29-31,37,39]$. The mechanism for ER downregulation by endocrine therapy is unclear. A previous study suggested that the effect was likely a post-transcriptional modification as there was no change of ER mRNA level[40]. On the other hand, the PR gene is an estrogenregulated gene, so drugs with endocrine activity would be expected to reduce PR expression. Indeed, post-treatment PR H-score of less than 10 was noted in four cases, all of whom were treated with letrozole.

In this cohort, five cases overexpressed Her2. There was no difference in Ki-67 reduction between Her2-positive and Her2-negative cases in this small study. The IMPACT trial which compared neoadjuvant tamoxifen to anastrozole in ER-positive invasive breast cancer has suggested that when compared to HER2-negative tumors, HER2-positive lesions tended to show less antiproliferative effect following endocrine treatment[27]. This effect may not have been detectable in this small cohort. However, the neoadjuvant therapy approach demonstrated in our study may provide an ideal model for addressing the impact of HER2 overexpression on the response to hormone therapy in DCIS in larger clinical trials.
Importantly, two of the 23 patients had invasive ductal carcinoma in addition to DCIS in post-treatment excision specimens. One of the invasive carcinomas was HER2positive, as was its associated DCIS. In both cases, the invasive tumors were hormone receptor-positive. The invasive carcinoma was likely present at initial diagnosis, but the focus missed due to sampling error. The rate of invasive cancer seen in our study is somewhat lower than reported rates of upstaging to invasive cancer (20-25\%) in patients with DCIS only on core biopsy[41,42]. This may reflect a higher degree scrutiny for invasive cancer for those patients enrolling on this study, as assessed on both clinical examination and radiographic work-up. Both patients with invasive cancer underwent clinical examination, mammography, and MRI prior to study entry, none of which were suspicious for invasive cancer. In the current study, this would not have been expected to impact patient outcome as all patients had ER-positive disease and definitive surgery was performed in all but two patients who declined surgery. Thus essentially, these postmenopausal patients were treated with a standard course of neoadjuvant aromatase inhibitor therapy. However, the risk of invasive disease not detected at stereotactic core biopsy is an important consideration for future trials of non-operative treatment for preinvasive disease, and underscores the importance of patient selection in the design of preoperative clinical trials in DCIS. Current efforts, including some in our own group, are focusing on efforts to improve discrimination of invasive cancer and DCIS on MRI by optimizing both image acquisition and software analysis technology to address this important need.

Finally, it is important to note that any studies seeking to evaluate non-surgical alternatives for what is currently a surgically treated disease will encounter challenges in study design, recruitment and analysis. Since DCIS is a noninvasive condition, it presents an ideal opportunity to study those patients at low risk of cancer progression, but clinical trial designs must be take into account the risk for invasive cancer, as discussed above, as well as patient acceptance of new treatment approaches, and must remain cognizant of the difficulties inherent in prospective randomization of patients between medical and surgical treatment alternatives. Many of these barriers will be overcome with greater patient and provider education.

\section{Conclusion}

In conclusion, preoperative hormone therapy for DCIS was feasible and well tolerated, and the effect of neoadjuvant hormone therapy in the setting of DCIS was associated with significant changes in both morphology and biomarker expression. The reduction of proliferation confirms that systemically administered drug delivery to intraductal lesions is sufficient to exert a favorable bio- 
logic effect. Whether this effect is sufficient to prevent invasive progression in the long-term is an important and compelling subject for additional research. However, our study opens the door for future trials evaluating the potential role of non-operative options for DCIS. The neoadjuvant approach described in our study provides a framework for evaluating the response of DCIS to preoperative therapies currently, including trials of agents targeting ER-negative disease. Such studies will yield important insight about the mechanisms involved in breast cancer progression and will thus inform the design of more targeted prevention studies. Although preliminary, our findings can initiate the dialogue for consideration of primary hormone therapy alone for DCIS in a select population of patients. As a follow-up to this current study, we are planning a multi-center clinical trial comparing longer intervals of letrozole treatment in postmenopausal women with ER-positive DCIS to further explore the acceptability, safety and efficacy of this approach.

\section{List of Abbreviations}

DCIS: Ductal carcinoma in situ; DFS: Disease-free survival; IHC: Immunohistochemistry; IRB: Institutional Review Board; H\&E: Hematoxylin and eosin; FISH: Fluorescent in-situ hybridization; ADH: Atypical ductal hyperplasia.

\section{Competing interests}

YYC, SD, JA, JL, RS, KC, VS, LJE, and FMW have no competing interests to declare. ESH - research grant from Novartis Corporation.

\section{Authors' contributions}

YYC, SD, JA, RS, FMW: IHC staining and scoring; YYC, SD, JA, JL, RS, KC, VS: data analysis and interpretation; YYC, FMW, LJE, ESH: study conception and design; FMW, ESH: data analysis; JL, VS, LJE, ESH: execution of clinical study; YYC, SD, FMW, ESH: manuscript drafting and preparation. All authors have read and approved the final manuscript.

\section{Additional material}

\section{Additional file 1}

Clinical parameters of study population. Clinical characteristics of study population.

Click here for file

[http://www.biomedcentral.com/content/supplementary/14712407-9-285-S1.doc]

\section{Additional file 2}

Immunohistochemistry. Comparison of pathologic features between preand post-treatment specimens.

Click here for file

[http://www.biomedcentral.com/content/supplementary/14712407-9-285-S2.doc]

\section{Additional file 3}

Representative low-power figures of ER, Ki67, CD68, and caspase 3 staining in low, intermediate, and high grade DCIS. All cases shown are post-treatment specimens. (A) ER strong staining (90\%) in low grade DCIS. (B) ER weak staining (25\%) in intermediate grade DCIS. (C) Ki67 strong staining (26\%) in high grade DCIS. (D) Ki67 weak staining (7\%) in intermediate grade DCIS. (E) CD68 strong staining (score 164) in intermediate grade DCIS. (F) CD68 weak staining (score 96) in intermediate grade DCIS. (G) caspase 3 strong staining $(8.8 \%)$ in high grade DCIS. (H) caspase 3 weak staining (1.6\%) in intermediate grade DCIS. Click here for file

[http://www.biomedcentral.com/content/supplementary/14712407-9-285-S3.doc]

\section{Acknowledgements}

We gratefully acknowledge the significant contributions of Sarah Nyante, Chrystal Wa, Laura Johnson, and Kaoru Itakura, for their excellent patient care as well as their dedication to the procurement and archiving of clinical information and pathologic specimens. We thank Amy Heinzerling for her expert preparation of the manuscript. This work was supported by NIH/ K23CA097I8I (ESH), the UCSF SPORE Clinical Investigator Research Program (P50 CA58207), and a research grant from Novartis Corporation. This work was presented in part at the $99^{\text {th }}$ Annual USCAP Meeting, Atlanta GA.

\section{References}

I. American Cancer Society: Breast Cancer Facts and Figures 2007-2008. Atlanta 2007.

2. Ernster VL, Barclay J, Kerlikowske K, Grady D, Henderson C: Incidence of and treatment for ductal carcinoma in situ of the breast [see comments]. Jama 1996, 275:913-918.

3. Ernster VL, Barclay J, Kerlikowske K, Wilkie H, Ballard-Barbash R: Mortality among women with ductal carcinoma in situ of the breast in the population-based surveillance, epidemiology and end results program. Arch Intern Med 2000, 160:953-958.

4. Nielsen M, Jensen J, Andersen J: Precancerous and cancerous breast lesions during lifetime and at autopsy. A study of $\mathbf{8 3}$ women. Cancer 1984, 54:612-615.

5. Bhathal PS, Brown RW, Lesueur GC, Russell IS: Frequency of benign and malignant breast lesions in 207 consecutive autopsies in Australian women. BrJ Cancer 1985, 5 I:27I-278.

6. Nielsen M, Thomsen JL, Primdahl S, Dyreborg U, Andersen JA: Breast cancer and atypia among young and middle-aged women: a study of 110 medicolegal autopsies. $\mathrm{Br} J$ Cancer 1987, 56:814-819.

7. Burstein HJ, Polyak K, Wong JS, Lester SC, Kaelin CM: Ductal carcinoma in situ of the breast. N Engl J Med 2004, 350: I 430-I 44 I.

8. Eusebi V, Feudale E, Foschini MP, Micheli A, Conti A, Riva C, Di Palma S, Rilke F: Long-term follow-up of in situ carcinoma of the breast. Semin Diagn Pathol 1994, 1 1:223-235.

9. Page DL, Dupont WD, Rogers LW, Jensen RA, Schuyler PA: Continued local recurrence of carcinoma 15-25 years after a diagnosis of low grade ductal carcinoma in situ of the breast treated only by biopsy. Cancer 1995, 76: I 197-1 200.

10. Collins LC, Tamimi RM, Baer HJ, Connolly JL, Colditz GA, Schnitt SJ: Outcome of patients with ductal carcinoma in situ untreated after diagnostic biopsy: results from the Nurses' Health Study. Cancer 2005, I03:1778-1784.

II. Erbas B, Provenzano E, Armes J, Gertig D: The natural history of ductal carcinoma in situ of the breast: a review. Breast Cancer Res Treat 2006, 97: I35- I 44.

12. Sanders ME, Schuyler PA, Dupont WD, Page DL: The natural history of low-grade ductal carcinoma in situ of the breast in women treated by biopsy only revealed over 30 years of long-term follow-up. Cancer 2005, 103:248I-2484.

13. Fisher B, Costantino J, Redmond C, Poisson R, Bowman D, Couture J, Dimitrov NV, Wolmark N, Wickerham DL, Fisher ER, et al.: A randomized clinical trial evaluating tamoxifen in the treatment of patients with node-negative breast cancer who have 
estrogen-receptor-positive tumors. N Engl J Med 1989, 320:479-484.

14. Fisher B, Dignam J, Wolmark N, Wickerham DL, Fisher ER, Mamounas E, Smith R, Begovic M, Dimitrov NV, Margolese RG, Kardinal CG, Kavanah MT, Fehrenbacher L, Oishi RH: Tamoxifen in treatment of intraductal breast cancer: National Surgical Adjuvant Breast and Bowel Project B-24 randomised controlled trial. Lancet 1999, 353:1993-2000.

15. Forbes JF, Cuzick J, Buzdar A, Howell A, Tobias JS, Baum M: Effect of anastrozole and tamoxifen as adjuvant treatment for early-stage breast cancer: 100-month analysis of the ATAC trial. Lancet Oncol 2008, 9:45-53.

16. Thurlimann B, Keshaviah A, Coates AS, Mouridsen H, Mauriac L, Forbes JF, Paridaens R, Castiglione-Gertsch M, Gelber RD, Rabaglio M, Smith I, Wardley A, Price KN, Goldhirsch A: A comparison of letrozole and tamoxifen in postmenopausal women with early breast cancer. N Engl J Med 2005, 353:2747-2757.

17. Page DL, Rogers LW: Combined histologic and cytologic criteria for the diagnosis of mammary atypical ductal hyperplasia. Hum Pathol 1992, 23:1095-1097.

18. World Health Organization Classification of Tumors. Pathology and Genetics of Tumors of the Breast and Female Genital Organs Lyon: IARC Press; 2004.

19. McCarty KS Jr, Miller LS, Cox EB, Konrath J, McCarty KS Sr: Estrogen receptor analyses. Correlation of biochemical and immunohistochemical methods using monoclonal antireceptor antibodies. Arch Pathol Lab Med 1985, 109:716-72I.

20. Snead DR, Bell JA, Dixon AR, Nicholson RI, Elston CW, Blamey RW, Ellis IO: Methodology of immunohistological detection of oestrogen receptor in human breast carcinoma in formalinfixed, paraffin-embedded tissue: a comparison with frozen section methodology. Histopathology 1993, 23:233-238.

21. Smith IE, Dowsett M, Ebbs SR, Dixon JM, Skene A, Blohmer JU, Ashley SE, Francis S, Boeddinghaus I, Walsh G: Neoadjuvant treatment of postmenopausal breast cancer with anastrozole, tamoxifen, or both in combination: the Immediate Preoperative Anastrozole, Tamoxifen, or Combined with Tamoxifen (IMPACT) multicenter double-blind randomized trial. J Clin Oncol 2005, 23:5108-5 II6.

22. Eiermann W, Paepke S, Appfelstaedt J, Llombart-Cussac A, Eremin J, Vinholes J, Mauriac L, Ellis M, Lassus M, Chaudri-Ross HA, Dugan M, Borgs M: Preoperative treatment of postmenopausal breast cancer patients with letrozole: A randomized double-blind multicenter study. Ann Oncol 200I, I 2:1527-I532.

23. Cataliotti L, Buzdar AU, Noguchi S, Bines J, Takatsuka Y, Petrakova K, Dube $\mathrm{P}$, de Oliveira CT: Comparison of anastrozole versus tamoxifen as preoperative therapy in postmenopausal women with hormone receptor-positive breast cancer: the Pre-Operative "Arimidex" Compared to Tamoxifen (PROACT) trial. Cancer 2006, 106:2095-2103.

24. Boland GP, McKeown A, Chan KC, Prasad R, Knox WF, Bundred NJ: Biological response to hormonal manipulation in oestrogen receptor positive ductal carcinoma in situ of the breast. $\mathrm{Br} J$ Cancer 2003, 89:277-283.

25. Clarke RB, Laidlaw IJ, Jones LJ, Howell A, Anderson E: Effect of tamoxifen on Ki67 labelling index in human breast tumours and its relationship to oestrogen and progesterone receptor status. Br J Cancer 1993, 67:606-6II.

26. Bajetta E, Celio L, Di Leo A, Bartoli C, Pilotti S, Leutner M, Bono A, Ferrari L, Buzzoni R, Zilembo N, De Candis D, Moglia D: Effects of short-term pre-operative tamoxifen on steroid receptor and Ki-67 expression in primary breast cancer: an immunocytochemical study. Int J Oncol I998, I 2:853-858.

27. Dowsett M, Ebbs SR, Dixon JM, Skene A, Griffith C, Boeddinghaus I, Salter J, Detre S, Hills M, Ashley S, Francis S, Walsh G, Smith IE: Biomarker changes during neoadjuvant anastrozole, tamoxifen, or the combination: influence of hormonal status and HER-2 in breast cancer \#I 50; a study from the IMPACT trialists. J Clin Oncol 2005, 23:2477-2492.

28. Dowsett M, Smith IE, Ebbs SR, Dixon JM, Skene A, Griffith C, Boeddinghaus I, Salter J, Detre S, Hills M, Ashley S, Francis S, Walsh G: Short-term changes in $\mathrm{Ki}-67$ during neoadjuvant treatment of primary breast cancer with anastrozole or tamoxifen alone or combined correlate with recurrence-free survival. Clin Cancer Res 2005, I I:95 Is-958s.

29. Harper-Wynne CL, Sacks NP, Shenton K, MacNeill FA, Sauven $P$, Laidlaw IJ, Rayter Z, Miall S, Howes A, Salter J, Hills MJ, Lowe FM,
A'Hern R, Nasiri N, Doody D, lqbal J, Dowsett M: Comparison of the systemic and intratumoral effects of tamoxifen and the aromatase inhibitor vorozole in postmenopausal patients with primary breast cancer. J Clin Oncol 2002, 20:1026-1035.

30. Miller WR, Dixon JM, Cameron DA, Anderson TJ: Biological and clinical effects of aromatase inhibitors in neoadjuvant therapy. I Steroid Biochem Mol Biol 200I, 79: 103-107.

31. Robertson JF, Nicholson RI, Bundred NJ, Anderson E, Rayter Z, Dowsett M, Fox JN, Gee JM, Webster A, Wakeling AE, Morris C, Dixon M: Comparison of the short-term biological effects of 7alpha-[9-(4,4,5,5,5-pentafluoropentylsulfinyl)-nonyl] estra1,3,5, (10)-triene-3,17beta-diol (Faslodex) versus tamoxifen in postmenopausal women with primary breast cancer. Cancer Res 200I, 61:6739-6746.

32. Dixon JM, Faratian D, White S, Renshaw L, Murray J, Young $O$ Macaskill EJ, Williams L, Thomas J, Evans DB: DCIS and aromatase inhibitors. J Steroid Biochem Mol Biol 2007, 106:173-179.

33. Mandlekar S, Yu R, Tan TH, Kong AN: Activation of caspase-3 and c-Jun NH2-terminal kinase-I signaling pathways in tamoxifen-induced apoptosis of human breast cancer cells. Cancer Res 2000, 60:5995-6000.

34. Obrero M, Yu DV, Shapiro DJ: Estrogen receptor-dependent and estrogen receptor-independent pathways for tamoxifen and 4-hydroxytamoxifen-induced programmed cell death. J Biol Chem 2002, 277:45695-45703.

35. Gandhi A, Holland PA, Knox WF, Potten CS, Bundred NJ: Effects of a pure antiestrogen on apoptosis and proliferation within human breast ductal carcinoma in situ. Cancer Res 2000 , 60:4284-4288.

36. Ellis PA, Saccani-Jotti G, Clarke R, Johnston SR, Anderson E, Howell A, A'Hern R, Salter J, Detre S, Nicholson R, Robertson J, Smith IE, Dowsett $\mathrm{M}$ : Induction of apoptosis by tamoxifen and $\mathrm{ICl}$ 182780 in primary breast cancer. Int J Cancer 1997, 72:608-6I3.

37. Dowsett M, Bundred NJ, Decensi A, Sainsbury RC, Lu Y, Hills MJ, Cohen FJ, Veronesi P, O'Brien ME, Scott T, Muchmore DB: Effect of raloxifene on breast cancer cell $\mathrm{Ki} 67$ and apoptosis: a doubleblind, placebo-controlled, randomized clinical trial in postmenopausal patients. Cancer Epidemiol Biomarkers Prev 200I, 10:961-966.

38. Rouanet P, Linares-Cruz G, Dravet F, Poujol S, Gourgou S, SimonyLafontaine J, Grenier J, Kramar A, Girault J, Le Nestour E, Maudelonde T: Neoadjuvant percutaneous 4-hydroxytamoxifen decreases breast tumoral cell proliferation: a prospective controlled randomized study comparing three doses of 4-hydroxytamoxifen gel to oral tamoxifen. I Clin Oncol 2005, 23:2980-2987.

39. Chang J, Powles TJ, Allred DC, Ashley SE, Makris A, Gregory RK, Osborne CK, Dowsett M: Prediction of clinical outcome from primary tamoxifen by expression of biologic markers in breast cancer patients. Clin Cancer Res 2000, 6:616-62I.

40. McClelland RA, Manning DL, Gee JM, Anderson E, Clarke R, Howell A, Dowsett M, Robertson JF, Blamey RW, Wakeling AE, Nicholson RI: Effects of short-term antiestrogen treatment of primary breast cancer on estrogen receptor mRNA and protein expression and on estrogen-regulated genes. Breast Cancer Res Treat 1996, 41:31-41.

4I. Lee CH, Carter D, Philpotts LE, Couce ME, Horvath LJ, Lange RC, Tocino I: Ductal carcinoma in situ diagnosed with stereotactic core needle biopsy: can invasion be predicted? Radiology 2000, 217:466-470.

42. Jackman RJ, Burbank F, Parker SH, Evans WP 3rd, Lechner MC, Richardson TR, Smid AA, Borofsky HB, Lee CH, Goldstein HM, Schilling KJ, Wray AB, Brem RF, Helbich TH, Lehrer DE, Adler SJ: Stereotactic breast biopsy of nonpalpable lesions: determinants of ductal carcinoma in situ underestimation rates. Radiology 200I, 2 I 8:497-502

\section{Pre-publication history}

The pre-publication history for this paper can be accessed here:

\section{http://www.biomedcentral.com/1471-2407/9/285/pre} pub 